Diabetologia 10, 685-690(1974)

(C) by Springer-Verlag 1974

\title{
Characterization of Diabetes Mellitus in South African Hamsters (Mystromys albicaudatus*)
}

\author{
R.A. Stuhlman, P.K. Srivastava, G. Schmidt, M. L. Vorbeck, and J.F. Townsend \\ Departments of Laboratory Animal Medicine and Pathology School of Medicine, University of Missouri, Columbia, \\ Missouri 65201, USA
}

Summary. Spontaneous diabetes mellitus has been studied in Mystromys albicaudatus (South African hamster) at the University of Missouri, Columbia Medical Center since its original discovery here in 1969. Thus far, characteristics established as part of the diabetic syndrome in this species included hyperglycemia, glucosuria, ketonuria, polyuria, polyphagia and polydipsia with variations in age of onset, degree of severity, and rate of progression. In addition, decreased glucose tolerance has been demonstrated and statistical analyses of data collected indicated that diabetes mellitus in $M$. albi- caudatus has a polygenic, nonsex-linked mode of inheritance. - Histologic findings involving the pancreas, kidneys, and liver were found to be positively correlated to diagnostic parameters as well as to their degree of severity and duration. Pancreatic lesions included $\beta$-cell vacuolization, glycogen infiltration, nuclear pyknosis, margination of organelles, and $\beta$-cell death.

Key words: Diabetes mellitus, characterization, Mystromys, South African hamster.
The South African hamster or white-tailed rat, Mystromys albicaudatus, is a member of the order Rodentia, family Cricetidae and is the only recognized member of the subfamily Cricetinae in Africa [1]. Although Mystromys were first introduced into the laboratory in 1941 in Africa, it was not until 1962 that the first colony was established in the United States at the Armed Forces Institute of Pathology (A.F.I.P.) [2].

The University of Missouri Columbia Medical Center (U.M.C.M.C.) colony of Mystromys albicaudatus was started in April 1968 with six adult animals, plus six of their unweaned offspring, provided by the A.F.I.P. In February 1969, the colony contained 88 animals, and it was at this time that some animals were noted repeatedly to have excessively wet cages, which lead to a diagnosis of spontaneous diabetes mellitus [3]. After the original survey of the colony, involving 118 animals [3], systematical studies were undertaken to define and characterize the syndrome of spontaneous diabetes mellitus in the colony [4-9].

Since 1968 , over 850 progeny have been weaned in the U.M.C.M.C.colony. Nearly 400 of these are still living, of which 140 are over 40 months of age. The biology and husbandry of Mystromys have been documented in detail $[2,5,10]$.

\section{Metabolic Studies}

Blood samples were collected from the tails of animals that were either non-fasted or fasted $5-7 \mathrm{~h}$ [5], using a specifically designed vacuum-tube apparatus [11]. Serum glucose values were initially determined

* The research was supported in part by USPHS GRANT 5-TO-1-FR05006 and VA Research Grant No. 8512-01 by the total reduction ferric cyanide autoanalyzer technique ${ }^{1}$. However, since May 1972, the hexokinase microtechnique for D-glucose ${ }^{2}$ has been employed. Values obtained with the autoanalyzer technique ranged from 60 to $2300 \mathrm{mg} / 100 \mathrm{ml}$ and those obtained with the hexokinase technique have ranged from 40 to $1000 \mathrm{mg} / 100 \mathrm{ml}$. Using data from our studies $[4,5]$ as well as data from other rodent models of diabetes [12-15], hyperglycemia in Mystromys was established as:

a) a nonfasted serum glucose value greater than $169 \mathrm{mg} / 100 \mathrm{ml}$ by autoanalyzer or $152 \mathrm{mg} / 100 \mathrm{ml}$ by hexokinase, or

b) a 5 to $7 \mathrm{~h}$ fasted value greater than $150 \mathrm{mg} / 100 \mathrm{ml}$ by autoanalyzer or $135 \mathrm{mg} / 100 \mathrm{ml}$ by hexokinase.

Hyperglycemia has been the most consistent characteristic of diabetes mellitus in our animals and is not affected by age, sex, or inbreeding [5] nor is it related to obesity $[3,5,7]$. However, it has varied in incidence, age of onset, degree of severity, and rate of progression as well as repeatability within individual animals. Table 1 depicts the variations in incidence of hyperglycemia with time and lists the April 1974 data in greater detail, illustrating that some variation in incidence may be the result of differences in exactly what is being reported as well as in what frame of reference. However, variations in incidence will occur, and are to be expected, when the number of reproducing diabetic and nondiabetic adults continually varies because of the need to determine whether or not the disease could be reproduced. Also, variation in age of onset will tend to increase the incidence of

1 Modified Method of W.S. Hoffman, J. biol. Chem. 120 (1937) as published by Technicon (1965).

2 Eskalab Instruction Manual, Smith Kline and French: Palo Alto, California. 1962. 
hyperglycemia as the number of older animals increases.

In some Mystromys, hyperglycemia is well established by four months of age $[3,5]$, while others have been various ages up to 40 months old before hyperglycemia was detected with the animals being normoglycemic on as many as 7 previous occasions. The majority of hyperglycemic values have been below $300 \mathrm{mg} / 100 \mathrm{ml}$. However, 67 higher values have been recorded with $2300 \mathrm{mg} / 100 \mathrm{ml}$ being the highest serum glucose value thus far determined. These high values have been consistently indicative of severe, rapidly progressing diabetes mellitus whereas the less extreme hyperglycemic values have not been as predictable weight; and 6. depression, debilitation, lethargy, ketoacidosis, coma and death usually within two months after the onset of signs if not treated. This form of the disease has been seen in less than $10 \%$ of the diabetic Mystromys.

Table 2 presents data on glycosuria and ketonuria in chronically diabetic animals as of April, 1974. In 251 males with chronic diabetes, $72 \%$ have been glucosuric $(80 \%$ of these intermittently) and $96 \%$ ketonuric $(39 \%$ of these intermittently). In 277 females with chronic diabetes, $29 \%$ have been glucosuric $(87 \%$ of these intermittently) and $29 \%$ ketonuric $(94 \%$ of these intermittently). However, hyperglycemia, glucosuria, and ketonuria have not always

Table 1. Variations in incidence of hyperglycemia in Mystromys

\begin{tabular}{llllll}
\hline & Jul. '69a & Nov. '69 & Mar. '70 & Mar. '73b & Apr. ${ }^{\text {b }} 74^{\mathrm{a}}$ \\
\hline Hyper F & $12 \%(5$ of 47$)$ & $31 \%(29$ of 93$)$ & $48 \%(59$ of 123$)$ & $50 \%(182$ of 364$)$ & $74 \%(288$ of 389$)$ \\
Hyper M & $30 \%(21$ of 71$)$ & $33 \%(39$ of 118$)$ & $54 \%(85$ of 158$)$ & $49 \%(205$ of 418$)$ & $69 \%(297$ of 430$)$ \\
Total Hyper & $22 \%(26$ of 118$)$ & $32 \%(68$ to 211$)$ & $51 \%(144$ of 281$)$ & $49 \%(387$ of 782$)$ & $71 \%(585$ of 819$)$ \\
\hline
\end{tabular}

April 1974: Details of hyperglycemia

\begin{tabular}{|c|c|c|}
\hline & 288 of 389 females $(74 \%)$ & 297 of 430 males $(69 \%)$ \\
\hline Repeated ${ }^{c}$ & $\begin{array}{l}195 \text { of } 389(50 \%) \\
195 \text { of } 288(68 \%) \\
195 \text { of } 585(33 \%)\end{array}$ & $\begin{array}{l}194 \text { of } 430(45 \%) \\
194 \text { of } 297(65 \%) \\
194 \text { of } 585(33 \%)\end{array}$ \\
\hline Alwaysd & 100 of $288(35 \%)$ & 83 of $297(28 \%)$ \\
\hline Classice $^{e}$ & 11 of $288 \quad(4 \%)$ & 46 of $297(15 \%)$ \\
\hline
\end{tabular}

a At least one hyperglycemic value

b Repeated hyperglycemic values

c Two or more hyperglycemic values

d All glucose determinations hyperglycemic (includes classic)

e Same as a but also all urinalyses detect glycosuria and ketonuria

Table 2. Glycosuria and ketonuria in Mystromys with chronic diabetes

\begin{tabular}{lllll}
\hline Number & Glycosuric & Intermittent & Ketonuric & Intermittent \\
\hline 251 males & 181 & 144 & 242 & 94 \\
277 females & 79 & 69 & 81 & 76 \\
\hline
\end{tabular}

as indicators of degree of severity or rate of progression of the disease. In addition, nearly $56 \%$ of the males and $45 \%$ of the females have demonstrated intermittent hyperglycemia as defined by one or more normoglycemic determination following recorded hyperglycemia.

Urinalyses consisted of measuring the volume of 16 to $20 \mathrm{~h}$ samples collected overnight and detecting the presence of glucose and/or ketones by the LabStix ${ }^{\circledR}$ method. The classic, severe, rapidly progressing, acute form of diabetes in Mystromys was characterized by: 1. polyuria and polydipsia - a $400 \%$ to $2000 \%$ increase; 2. glycosuria $-0.4 \mathrm{~g} / 100 \mathrm{ml}$ or more consistently; 3 . ketonuria - consistent moderate to large reading on the Lab-Stix; 4. extreme hyperglycemia - values continuously above $400 \mathrm{mg} / 100 \mathrm{ml}$; 5. polyphagia with severe weight loss - up to several times normal food intake while losing $50 \%$ of body occurred together, nor have the degrees of severity of each always reflected the others.

Therefore, while polyuria, polydipsia, glucosuria and ketonuria were the first observed characteristics of diabetes in Mystromys [3], the consistency and degree of severity with which they have occurred in the presence of other, more definitive characteristics of the disease have varied greatly. Similar variations have been reported in humans [16-20].

Decreased glucose tolerance has been demonstrated in diabetic Mystromys and the details reported elsewhere [9].

\section{Morphologic Studies}

Pancreatic islet cells from normoglycemic Mystromys showed distinct cell outlines and contained no 
cytoplasmic vacuoles or PAS positive materials (Fig. 1). The morphologic alterations described in the $\beta$-cells of a few selected, hyperglycemic Mystromys consisted of progressive vacuolization, glycogen infiltration demonstrated by PAS-hemotoxylin stained sections before and after diastase digestion, and nuclear pylnnosis (Fig. 2) [3, 4]. A comparison of the degree of severity of pancreatic islet lesions at the light microscopy level to the serum glucose means in 127 animals gave statistical validity to the decision to use nonfasted serum glucose values greater than $169 \mathrm{mg} / 100 \mathrm{ml}$ as denoting hyperglycemia [7]. tromys and to study the somatic chromosomes. Based on data accumulated in our studies $[3-5,7,9]$, animals were categorized as:

1. diabetic - repeated hyperglycemia and/or decreased glucose tolerance and/or characteristic pancreatic lesions;

2. suspect - multiple serum glucose determinations but only one instance of hyperglycemia with all other criteria normal; or

3. normal - for all criteria.

Comparison of the numbers of each phenotype

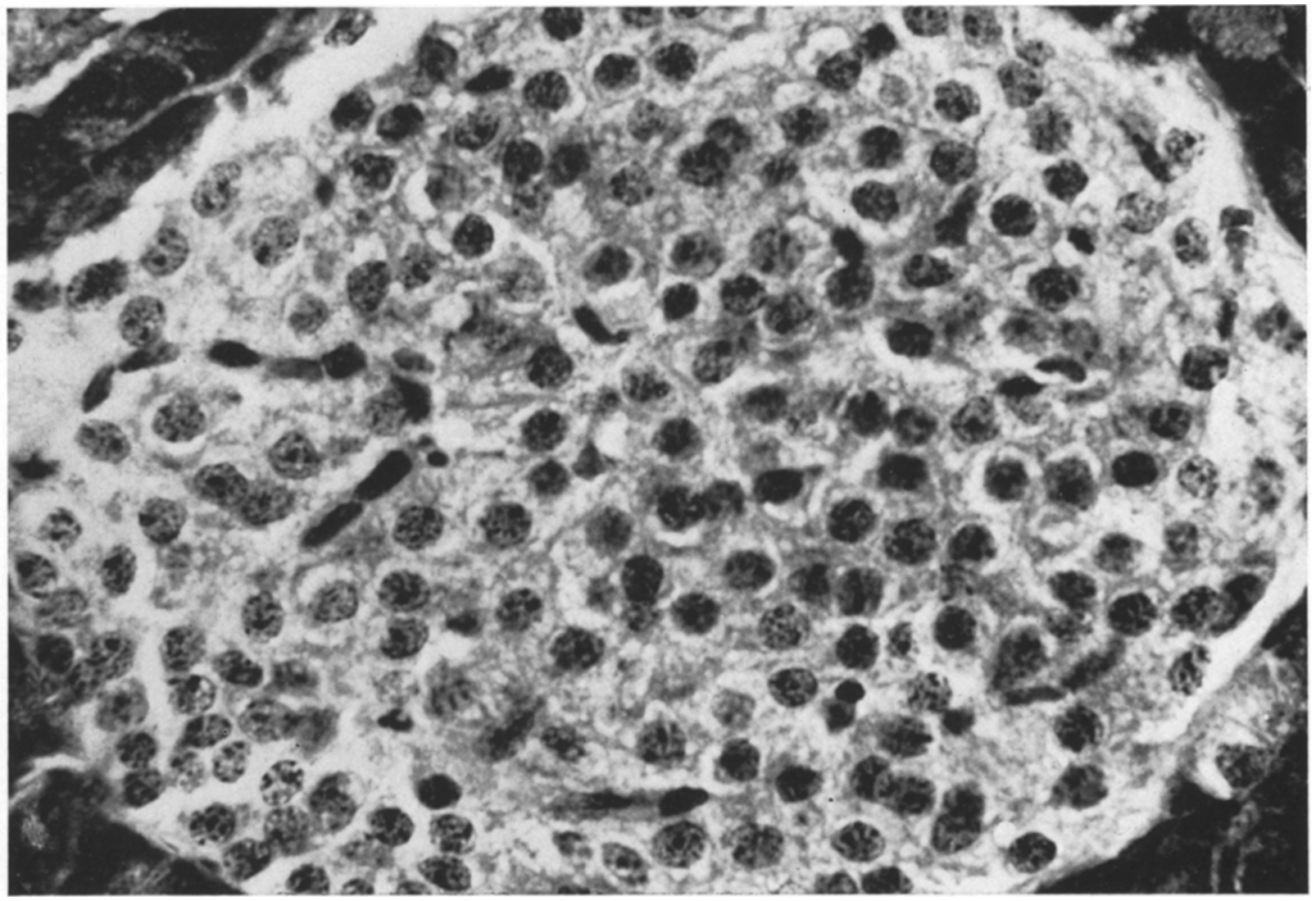

Fig. 1. Normal pancreatic islet (vascular spaces scattered throughout the islet are not degenerative changes). Hematoxylin and eosin stained $(x 1500)$

Additional morphologic studies have included electron microscopic analysis of the pancreatic islets [4], ultrastructures of hepatic mitochondria [6] and light microscopic evaluation of renal glomeruli [8]. The morphologic changes in the hepatic mitochondria were reflected by increased respiratory control ratios and elevated activity of $\beta$-hydroxybutyrate dehydrogenase [6].

\section{Genetic Studies}

These investigations were undertaken to determine the mode of inheritance of diabetes mellitus in Mys- born to the diabetic $\mathrm{x}$ diabetic and normal $\mathrm{x}$ normal matings resulted in a chi-square of 663.8 , thereby providing the criterion necessary to prove that heredity plays a significant role in the causation of diabetes mellitus in Mystramys.

The number of offspring, by sex, from each of the nine possible mating arrangements were analysed. There were no significant differences in the numbers of offspring per phenotype from the three reciprocal crosses, hence no sex-linkage. There was no significant difference in the numbers of each sex within the mating arrangements, hence no sex-control or sex-influence.

Statistical analyses of repeated serum glucose 
values from 620 Mystromys suggested a polygenic mode of inheritance of the disease in this species [5]. Further support was obtained when a midparent regression analysis yielded a heritability estimate of 0.391 , indicating a polygenic trait composed of small additive effects.

The chromosome complement of Mystromys from the U.M.C.M.C. colony was studied using heart blood culture [21] and our findings differ from the only other two reports, which also differ from each other, some was the longest submetacentric and the $Y$ was an acrocentric, ranking seventh in the order of decreasing size. Both of the two previous reports described the $\mathrm{Y}$ chromosome as the smallest of the complement. When the $\mathrm{Y}$ chromosome is not the smallest, its identification is equivocal. By analyzing the G-banding patterns (Fig. 4), it becomes obvious that the $X$ and $Y$ were the only nonhomologous pairs and that their banding patterns were unique. Therefore, we were reasonably sure about our identification of the sex chromosomes.

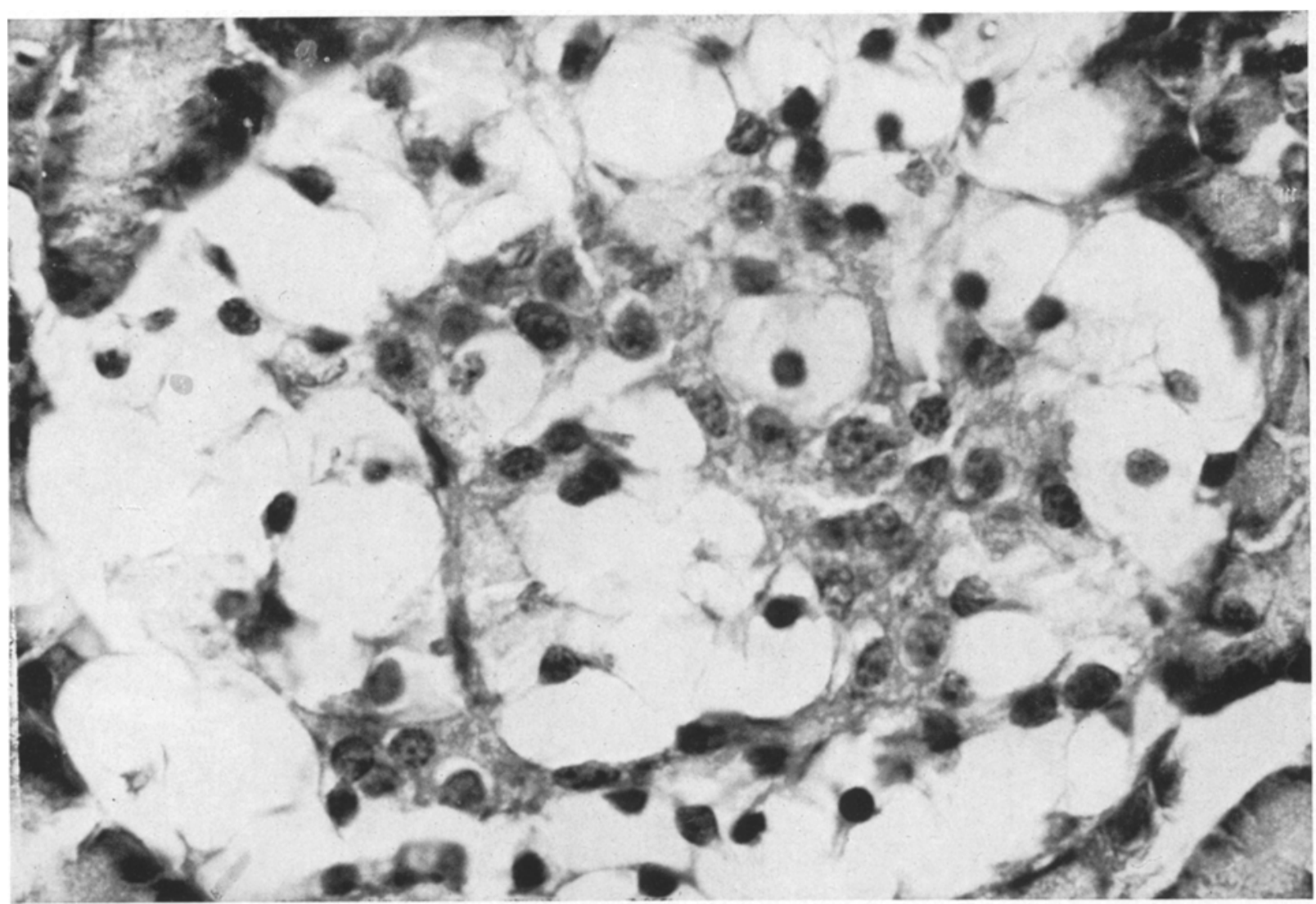

Fig. 2. Pancreatic islet with severe lesions (mostly vacuoles, glycogen infiltrated cells and pyknotic nuclei; few, if any, normal islet cells). Hematoxylin and eosin $(\times 1500)$

on the chromosomes of this species [22, 23]. A diploid number of 32 chromosomes was reported in all three publications but the descriptions of the chromosomes varied greatly. We arranged them in the order of decreasing length on the basis of form and size (Fig. 3). The autosome pairs numbered 1, 2, and 15 were submetacentric; $3,4,5,10,11,13$, and 14 were metacentric; and $6,7,8,9$, and 12 were acrocentric. Taitz [22] described all chromosome pairs as submetacentric. Hsu and Benirschke [23] did not provide a precise morphological description of individual chromosome pairs.

Sex chromosomes of the males were determined in our study by the process of elimination. The X chromo-

\section{Comments}

The data presented has demonstrated that spontaneous diabetes mellitus develops in the U.M.C.M.C. colony of Mystromys albicaudatus. The disease has a complex, diverse nature characterized by hyper-

Fig. 3. Somatic chromosomes of $M$. albicaudatus. The autosomes are arranged in decreasing order of length (pair 1 to 15). The sex chromosomes are in the bottom lime $(\times 2800)$

Fig. 4. G-banding patterns of a male $M$. albicaudatus. Chromosomes are in midprophase. Note the nonhomologous nature of the $X$ and $Y$ chromosomes $(X 3000)$ 
THE AUTOSOMES $a x$

1

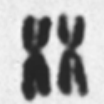

2

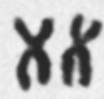

3

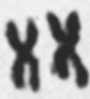

4
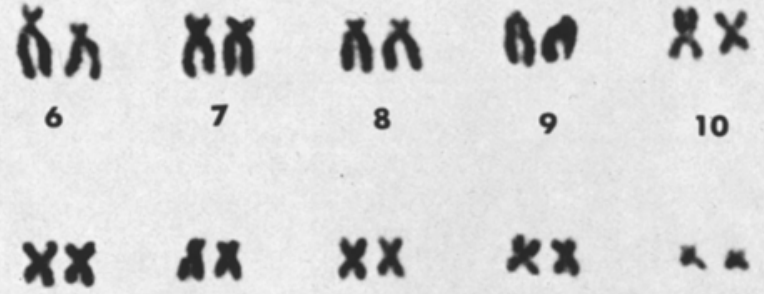

12
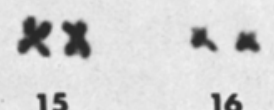

16
5

10

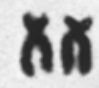

7

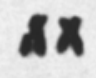

13

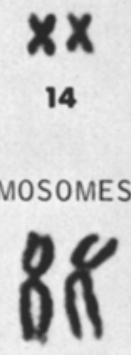

$\mathbf{X X}$

\section{SEX CHROMOSOMES}

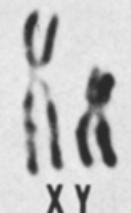

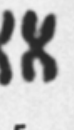

Fig. 3

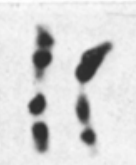

2
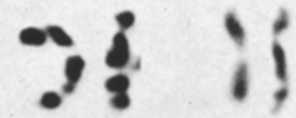

8
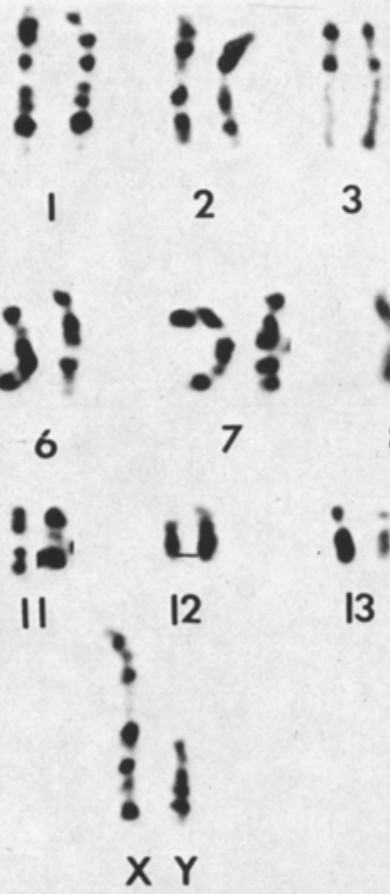

3

7

12

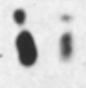

13
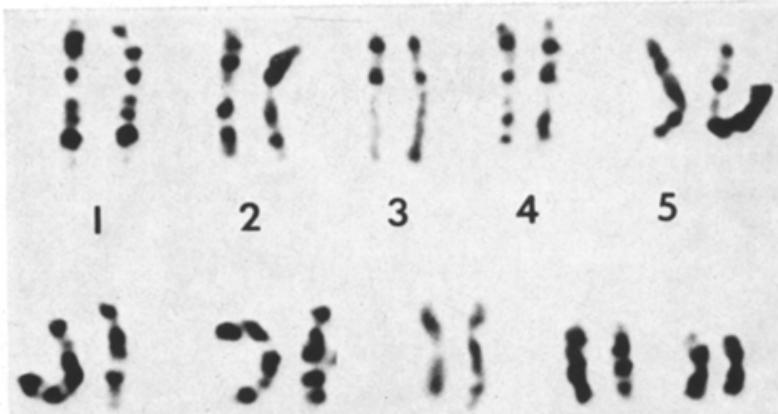

4

5
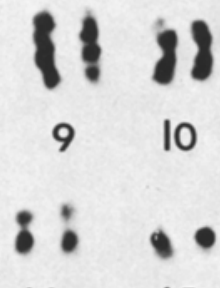

14

glycemia, glucosuria, ketonuria, polyuria, polydipsia, and polyphagia which are variable in age of onset, degree of severity, and rate of progression. In addition, decreased glucose tolerance occurs as part of the syndrome [9].

Histologic characteristics of this metabolic disorder include cytoplasmic vacuolization and glycogen infiltration, margination of organelles, and nuclear pyknosis of pancreatic islet cells $[3,4]$; capillary basement membrane thickening and mesangial proliferation in renal glomeruli [8]; generalized ballooning and vacuolization of hepatic nuclei and centrolobular fatty infiltration [6]; and hepatic mitochondrial enlargement with abnormal shapes [6]. The morphologic alterations of the hepatic mitochondria are reflected by increased respiratory control ratios and increased specific activity of $\beta$-hydroxybutyrate dehydrogenase [6].

Evaluation of the accumulated data indicates that spontaneous diabetes mellitus in $M$. albicaudatus is reproducible and has a polygenic, nonsex-linked mode of inheritance.

Acknowledgements. The authors are indebted to Drs. R.V. Brown, R.E. Doyle, J.A. Esterly, J.A. Goeken, K.L. Kraner, F.V. Lucas, A.P. Martin, L.R. Nelson, J.T. Packer, T. Riley, S.D. Rose, and H.J. Van Peenen for their permission to include some of the data in the collaborative studies, and to Janice Franz, Betty Bross, and John Gaughan for their technical assistance.

\section{References}

1. Ellerman, J.R. : Families and genera of living rodents. Vol. 2. London: Jarrold and Sons Ltd. 1941

2. Hall, A., Persing, R.L., White, D.C., Ricketts, R.'T.: Mystromys albicaudatus (the African white-tailed rat) as a laboratory species. Lab. Anim. Care 17, 180-187 (1967)

3. Packer, J.T., Kraner, K.L., Rose, S.D., Stuhlman, R.A., Nelson, L.R.: Diabetes mellitus in Mystromys albicaudatus. Arch. Path. 89, 410-415 (1970)

4. Goeken, J.A., Packer, J.T., Rose, S.D., Stuhlman, R.A.: Structure of the islets of Langerhans: Pathological studies in normal and diabetic Mystromys albicaudatus. Arch. Path. 93, 123-129 (1972)

5. Stuhlman, R.A., Packer, J.T., Doyle, R.E.: Spontaneous diabetes mellitus in $M$ ystromys albicaudatus: Repeated glucose values from 620 animals. Diabetes 21, 715-721 (1972)

6. Schmidt, G., Martin, A.P., Stuhlman, R.A., Townsend, J.F., Lucas, F.V., Vorbeck, M.L.: Evaluation of hepatic mitochondrial function in the spontaneous diabetic Mystromys albicaudatus. Lab. Invest. 30, $451-457$ (1974)

7. Stuhlman, R.A., Packer, J.T., Doyle, R.E., Brown, R.V., Townsend, J.F.: Relationship between pancreatic lesions and serum glucose values in Mystromys albicaudatus. Lab. Anim. Sci. (in press)

8. Riley, T., Stuhlman, R.A., Van Peenen, H.J., Esterly, J.A., Townsend, J.F.: Glomerular lesions of diabetes mellitus in Mystromys albicaudatus. Arch. Path. (in press)

9. Stuhlman, R.A., Packer, J.T., Doyle, R.E., Townsend, J.F.: Glucose tolerance test results from

Fig. 4 
Mystromys albicaudatus (white-tailed rat). Submitted to Lab. Anim. Sci. for publication

10. Stuhlman, R.A.: The genetic mode of transmission of spontaneous diabetes mellitus in Mystromys albi. caudatus. University of Missouri, Columbia: M.S. thesis, 1971

11. Stuhlman, R.A., Packer, J.T., Rose, S.D.: Repeated blood sampling of Mystromys albicaudatus (whitetailed rat). Lab. Anim. Sci. 22, 268-270 (1972)

12. Bleisch, V.R., Mayer, J., Dickie, M.M.: Familial diabetes mellitus in mice, associated with insulin resistance, obesity and hyperplasia of the islets of Langerhans. Amer. J. Path. 28, 369-380 (1952)

13. Coleman, D.L., Hummel, K.P.: Studies with the mutation, diabetes, in the mouse. Diabetologia 3, $238-248$ (1967)

14. Gerritsen, G.C., Dulin, W.E.: Characterization of diabetes in the Chinese hamster. Diabetologia 3, $74-84(1967)$

15. Hummel, K.P., Dickie, M.M., Coleman, D.L.: Diabetes, a new mutation in the mouse. Science 153, $1127-1128(1966)$

16. Alexander, R.W.: Diabetes mellitus - current criteria for laboratory diagnosis. Calif. Med. 110, 107-113 (1969)

17. Elsom, K.O., Ipsen, J., Clark, T.W., Talerico, L., Yanagawa, H.: Physician's use of objective data in clinical diag'noses. J. Amer. med. Ass. 201, 519-526 (1967)

18. Jackson, W.P. U., Marine, N., Vinik, A. I. : Significance of glycosuria. Lancet 1968 I, 933-936

19. Kent, G.T., Leonards, J.R.: Analysis of tests for diabetes in 250,000 persons screened for diabetes using finger blood after a carbohydrate load. Diabetes 17, $274-280(1968)$

20. Winegrad, A. I., Burden, C. L.: L-Xylulose metabolism in diabetes mellitus. New Engl. J. Med. 274, 298-305 (1966)

21. Srivastava, P.K., Townsend, J.F., Stuhlman, R.A., Lucas, F.V.: Somatic chromosomes of Mystromys albicaudatus. J. Heredity (in press)

22. Taitz, L.S.: Chromosomes of the white-tailed rat (Mystromys albicaudatus, Wagner, 1841). S. Afr. J. Sci. 51, 143-148 (1954)

23. Hsu, T.C., Benirschke, K.: An atlas of mammalian chromosomes. Vol. 2, Folia 60. New York: SpringerVerlag 1968

Robert A. Stuhlman, D.V.M., M.S.

Depts. of Laboratory Animal

Medicine and Pathology

University of Missouri

Columbia, Missouri 65201

USA. 